OPEN ACCESS

Edited by:

Billy Sperlich,

University of Würzburg, Germany

Reviewed by: Fabio Rubens Serpiello, Victoria University, Australia, Australia

Pedro Jiménez Reyes, Universidad Católica San Antonio de Murcia, Spain

*Correspondence: Carlos Balsalobre-Fernández carlos.balsalobre@icloud.com

Specialty section This article was submitted to Exercise Physiology a section of the journal Frontiers in Physiology

Received: 16 March 2017 Accepted: 16 August 2017 Published: 28 August 2017

Citation:

Balsalobre-Fernández $C$

Marchante D, Baz-Valle E,

Alonso-Molero I, Jiménez SL and Muñóz-López M (2017) Analysis of

Wearable and Smartphone-Based Technologies for the Measurement of Barbell Velocity in Different Resistance Training Exercises. Front. Physiol. 8:649 doi: 10.3389/fphys.2017.00649

\section{Analysis of Wearable and Smartphone-Based Technologies for the Measurement of Barbell Velocity in Different Resistance Training Exercises}

\author{
Carlos Balsalobre-Fernández ${ }^{1,2 *}$, David Marchante ${ }^{2}$, Eneko Baz-Valle ${ }^{2}$, \\ Iván Alonso-Molero ${ }^{2}$, Sergio L. Jiménez ${ }^{1}$ and Mario Muñóz-López ${ }^{2}$
}

${ }^{1}$ School of Sports Science, European University of Madrid, Madrid, Spain, ${ }^{2}$ Department of Research and Development, PowerExplosive Center, Madrid, Spain

The purpose of this study was to analyze the validity, reliability, and accuracy of new wearable and smartphone-based technology for the measurement of barbell velocity in resistance training exercises. To do this, 10 highly trained powerlifters (age $=26.1$ \pm 3.9 years) performed 11 repetitions with loads ranging $50-100 \%$ of the 1 -Repetition maximum in the bench-press, full-squat, and hip-thrust exercises while barbell velocity was simultaneously measured using a linear transducer (LT), two Beast wearable devices (one placed on the subjects' wrist -BW-, and the other one directly attached to the barbell -BB-) and the iOS PowerLift app. Results showed a high correlation between the LT and BW $\left(r=0.94-0.98\right.$, SEE $\left.=0.04-0.07 \mathrm{~m}^{\bullet} \mathrm{s}^{-1}\right)$, BB $(r=0.97-0.98$, SEE $\left.=0.04-0.05 \mathrm{~m}^{\bullet} \mathrm{s}^{-1}\right)$, and the PowerLift app $(r=0.97-0.98$, SEE $=0.03-0.05$ $\mathrm{m} \bullet \mathrm{s}^{-1}$ ) for the measurement of barbell velocity in the three exercises. Paired samples $T$-test revealed systematic biases between the LT and BW, BB and the app in the hip-thrust, between the LT and BW in the full-squat and between the LT and BB in the bench-press exercise $(p<0.001)$. Moreover, the analysis of the linear regression on the Bland-Altman plots showed that the differences between the $L T$ and BW $\left(R^{2}=0.004-0.03\right)$, BB $\left(R^{2}=0.007-0.01\right)$, and the app $\left(R^{2}=0.001-0.03\right)$ were similar across the whole range of velocities analyzed. Finally, the reliability of the BW (ICC $=0.910-0.988)$, BB (ICC $=0.922-0.990)$, and the app (ICC $=0.928-0.989)$ for the measurement of the two repetitions performed with each load were almost the same than that observed with the LT (ICC $=0.937-0.990)$. Both the Beast wearable device and the PowerLift app were highly valid, reliable, and accurate for the measurement of barbell velocity in the bench-press, full-squat, and hip-thrust exercises. These results could have potential practical applications for strength and conditioning coaches who wish to measure barbell velocity during resistance training.

Keywords: monitoring, biomechanics, technology, strength, validation 


\section{INTRODUCTION}

Quantifying and monitoring intensity is a key part when designing resistance training programs (Folland and Williams, 2007; Borresen and Lambert, 2009; Tillin and Folland, 2014); in fact, training intensity is considered the most important variable to produce the desired neuromuscular adaptations (Folland and Williams, 2007). Several approaches have been used for decades to monitor the intensity during resistance training (GonzalezBadillo and Sánchez-Medina, 2010; Zourdos et al., 2015; Naclerio and Larumbe-Zabala, 2016), with the 1-Repetition maximum (1RM) being the most widely used in the field of strength and conditioning (Buckner et al., 2017). To measure the 1-RM, the athlete needs to perform a maximal lift with a load that can be moved just once; therefore, given the extreme effort it represents, different strategies have emerged to indirectly estimate the 1-RM in a less demanding way (Kravitz et al., 2003; Robertson et al., 2008; Jidovtseff et al., 2011; Picerno et al., 2016). Among them, the measurement of the velocity at which the barbell is moved in the concentric phase on different resistance exercises has been shown to provide accurate, indirect estimations of the 1-RM without the need to perform a maximal lift (Gonzalez-Badillo and Sánchez-Medina, 2010; Jidovtseff et al., 2011; Picerno et al., 2016; Muñoz-López et al., 2017). These studies, mainly conducted within the last decade, are based on the very high correlation $\left(R^{2}>0.97\right)$ observed between the load (in terms of \%1-RM) and the mean velocity at which each load is lifted (Gonzalez-Badillo and Sánchez-Medina, 2010; Conceição et al., 2016; MuñozLópez et al., 2017). Thus, based on load-velocity profiles, the measurement of movement velocity during resistance training can be used to estimate 1-RM and each of its percentages, which could help adjusting training intensity (Muñoz-López et al., 2017). Moreover, movement velocity during resistance training has shown to provide accurate estimations of the degree of neuromuscular fatigue (Sánchez-Medina and Gonzalez-Badillo, 2011). For example, it has been observed that manipuling the percentage of velocity loss during the set influences the increases in strength and hypertrophy (Pareja-Blanco et al., 2016), with lower percentages of loss being associated with greater performance improvements and less muscle mass gains. Therefore, analyzing the drop of velocity within the sets could be used to optimize the adaptations to resistance training (SánchezMedina and Gonzalez-Badillo, 2011; Tufano et al., 2016).

To measure barbell velocity, different technologies, such as accelerometers, professional video systems or linear transducers (LT) have been used, with LT often considered the gold standard (Balsalobre-Fernández et al., 2016b, 2017; Banyard et al., 2016). An LT consists of a sensor with a cable that is attached to the barbell and measures barbell velocity by differentiating cable displacement with respect to time. However, many available LTs are still too expensive for many coaches ( $\sim$ US $\$ 2,000)$, which prevents its use outside professional clubs or sports sciences laboratories. For this reason, recent studies have analyzed the validity and reliability of more affordable technologies used to measure barbell velocity in resistance exercises, like high-speed cameras, smartphone apps, or wearable devices (BalsalobreFernández et al., 2016b, 2017; Sañudo et al., 2016). For example, an iOS app named PowerLift was recently validated for the measurement of barbell velocity with respect to a $1 \mathrm{kHz} \mathrm{LT}$ in the bench-press exercise (Balsalobre-Fernández et al., 2017). Specifically, the use of wearable devices in sports sciences is getting a lot of attention during the last years; in fact, "Wearable devices" is the \# 1 fitness trend in the 2017 edition of the American College of Sports Medicine survey (Thompson, 2016). However, the use of wearable devices for the measurement of physical performance is currently questioned (Halson, 2016), since just a few of the hundreds of models available are scientifically validated. For example, to the best of our knowledge, there is just one validated wearable device for the measurement of barbell velocity in resistance exercises (i.e., the PUSH band) (BalsalobreFernández et al., 2016b; Banyard et al., 2017). Thus, the purpose of the present study was to analyze the concurrent validity and reliability of a popular wearable device (i.e., the Beast sensor) for the measurement of barbell velocity in the full-squat, benchpress, and hip-thrust exercises. Additionally, we aimed to test if the positive results observed in the previously validation paper of the PowerLift iOS app (Balsalobre-Fernández et al., 2017) were confirmed in the full-squat and the hip-thrust exercises, since movement velocity can greatly vary between different exercises (Sánchez-Medina et al., 2014; Conceição et al., 2016).

\section{MATERIALS AND METHODS}

\section{Participants}

Ten highly-trained, competitive powerlifters at national and international events took part in this study $[N=10 ; 6$ men, 4 women; age $=26.1 \pm 3.9$ years, body mass index $=23.2$ $\pm 3.3 \mathrm{~kg} / \mathrm{m}^{2}, 1-\mathrm{RM}$ relative to body mass $(\mathrm{kg} / \mathrm{kg}): 1.93 \pm$ 0.5 -full-squat-, $1.3 \pm 0.5$-bench-press-, $2.9 \pm 0.7$-hipthrust-]. The study protocol complied with the Declaration of Helsinki for Human Experimentation and was approved by the ethics committee at the European University of Madrid, Spain. Written informed consent was obtained from each participant in advance.

\section{Experimental Design}

Participants performed six incremental sets until they reached their 1-Repetition maximum on the full-squat, bench-press, and hip-thrust exercises while mean barbell displacement velocity was measured with a Smartcoach Power Encoder linear transducer (LT), two Beast sensor wearable devices (one fixed to the barbell - $\mathrm{BB}-$, another one attached to a wrist-band that the participants wore on their right wrist -BW-) and the PowerLift iOS app simultaneously. Two repetitions were completed with the five initial sets (which corresponded approximately to 50, $60,70,80$, and $90 \%$ of the $1-\mathrm{RM}$ ), while one repetition was performed with the last set (i.e., the $100 \% 1-\mathrm{RM}$ ); therefore, 11 repetitions were measured for each participant and exercise for a total of 330 repetitions. Several statistical analyses were conducted in order to compare the velocities measured with $\mathrm{BB}, \mathrm{BW}$ and the app with those obtained with the LT. Finally, the load-velocity profiles (i.e., the slope, y-intercept and coefficient of determination of the regression line) computed with the loads used in the six incremental sets (in $\mathrm{kg}$ ) 
and its associated velocities measured with each device were compared.

\section{1-RM Incremental Test}

Participants were asked to perform three 1RM tests, one for each exercise (i.e., full squat, bench press, and hip-thrust), on three separate occasions interspersed with $48 \mathrm{~h}$ of passive rest. The $1-\mathrm{RM}$ incremental tests consisted of five sets with loads ranging $\sim 50-90 \%$ 1-RM, and one last set with the actual 1-RM. With the submaximal loads, participants performed two repetitions, while with the 1-RM they did just one. When participants were able to perform more than one repetition, an additional set with a heavier load was performed in order to reach the 1-RM. Sets were separated by $5 \mathrm{~min}$ of passive rest. Athletes were instructed to perform each repetition as fast as possible, and all of them were experts on the full-squat, bench-press, and hip-thrust exercises.

\section{Data Collection}

\section{Linear Transducer}

A SmartCoach Power Encoder (SmartCoach Europe, Stockholm, Sweden) linear transducer (LT) was considered as the criterion for the measurement of barbell displacement velocity in the present study. Mean velocity (in $\mathrm{m}^{-1}$ ) of each repetition was recorded at a sampling rate of $1 \mathrm{kHz}$ by attaching the cable of the LT to the barbell, aligned with the vertical axis as described by the manufacturer (i.e., perpendicular to the ground). Specifically, the cable was attached to the right end of the barbell, close to the weight plates. See Figure 1. Then, the LT was connected to the Smartcoach software 5.0.0 installed on a personal computer running the Windows 10 operating system, which provided mean velocity values in real time.

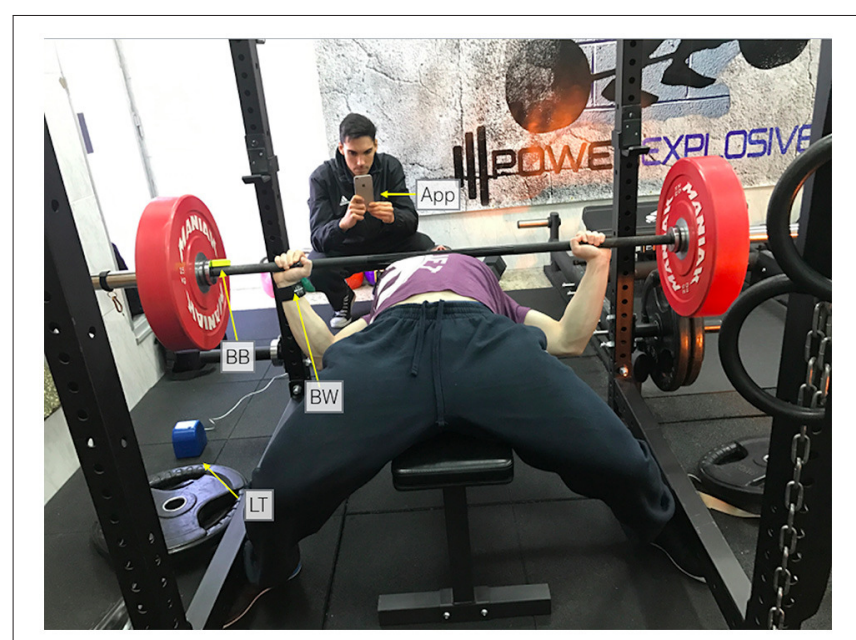

FIGURE 1 | Setup of the different devices during the measurement of barbell velocity in the bench-press exercise. App: PowerLift app; BW = Beast sensor (wrist); BB = Beast sensor (barbell); LT = linear transducer. Written informed consent was obtained from the two identifiable subjects for the publication of this picture.

\section{Beast Sensor Wearable Device}

The Beast sensor is a small device $\left(15.2 \mathrm{~cm}^{3}\right.$ of volume, 38 $\mathrm{g}$ of weight) which includes a 3 -axis accelerometer, gyroscope and magnetometer that measures velocity at a sampling rate of $50 \mathrm{~Hz}$. This wearable was designed to be fixed to a wristband that the athlete wears while training. Also, it can be positioned on to the barbell thanks to a built-in magnet. In order to test both configurations, two Beast sensors were used in the present study: one was directly fixed to the right end of the barbell (BB) and another one was placed in the right wrist of the participants using a wristband designed by the same manufacturer (BW). See Figure 1 for more details. Then, mean velocity (in $\bullet^{-1}$ ) of each repetition was transferred in real time via Bluetooth 4.0 LE to the Beast app for iOS v.2.2.3, which was installed on an iPhone 6 with iOS 10.2.1 operative system.

\section{PowerLift App}

Finally, the PowerLift v.4.0 iOS app, installed on an iPhone 6 running iOS 10.2.1 was used to measure barbell velocity in the three mentioned exercises as well. The app was designed to measure barbell velocity by video-recording the lift at slow motion thanks to the high-speed camera included in current iOS devices. Then, the app allowed a frame-by-frame inspection of the video to manually select the beginning and end of the movement, and therefore measure the time of the concentric phase of the lift. Finally, mean vertical barbell velocity (in møs ${ }^{-1}$ ) was computed as the range of motion (ROM) of the concentric phase of the exercise divided by the time of the lift. For the full-squat exercise, the ROM was calculated as the vertical difference between the height of the barbell with respect to the ground on the final position (i.e., knees extended) and the height of the barbell at the bottom position (i.e., thighs parallel to the ground). The beginning of the movement was considered as the first frame in which the barbell started to ascend vertically, while the end was considered as the first frame in which the barbell stopped that ascension. For the bench-press exercise, the ROM was calculated as the vertical distance between the barbell in the final position of the exercise (i.e., elbows fully extended) and the chest of the athlete. The beginning of the lift was considered as the first frame in which the barbell left the chest of the participant, while the end was considered as the first frame in which the barbell ended its vertical displacement. Finally, in the hip-thrust exercise, the ROM was calculated as the vertical distance between the bottom of the plate in the final position of the exercise (i.e., hip extended, thighs parallel to the ground) and the ground. The beginning of the movement was considered as the first frame in which the weight plates left the ground, while the end was considered as the first frame in which the vertical displacement of the barbell ends. To measure the ROM, an experienced researcher used a metric tape, while beginning and end frames were manually selected by an observer with experience in video analysis. All the videos were recorded and analyzed at 240 frames per second (FPS), at a quality of $720 \mathrm{p}$. 


\section{Statistical Analyses}

Several statistical analyses were used to test the accuracy, validity, and reliability of the Beast wearable device and the PowerLift app for the measurement of barbell velocity in the full-squat, bench-press and hip-thrust exercises in comparison with the gold standard (i.e., the LT).

\section{Accuracy}

Paired samples $t$-test and Bland-Altman plots were used to identify potential systematic bias, reported via mean absolute (in $\mathrm{m} / \mathrm{s}$ ) and relative (in \%) bias and standard deviations. Furthermore, standard error of estimate (SEE) was also used to report the typical error in the measurements in comparison with the LT.

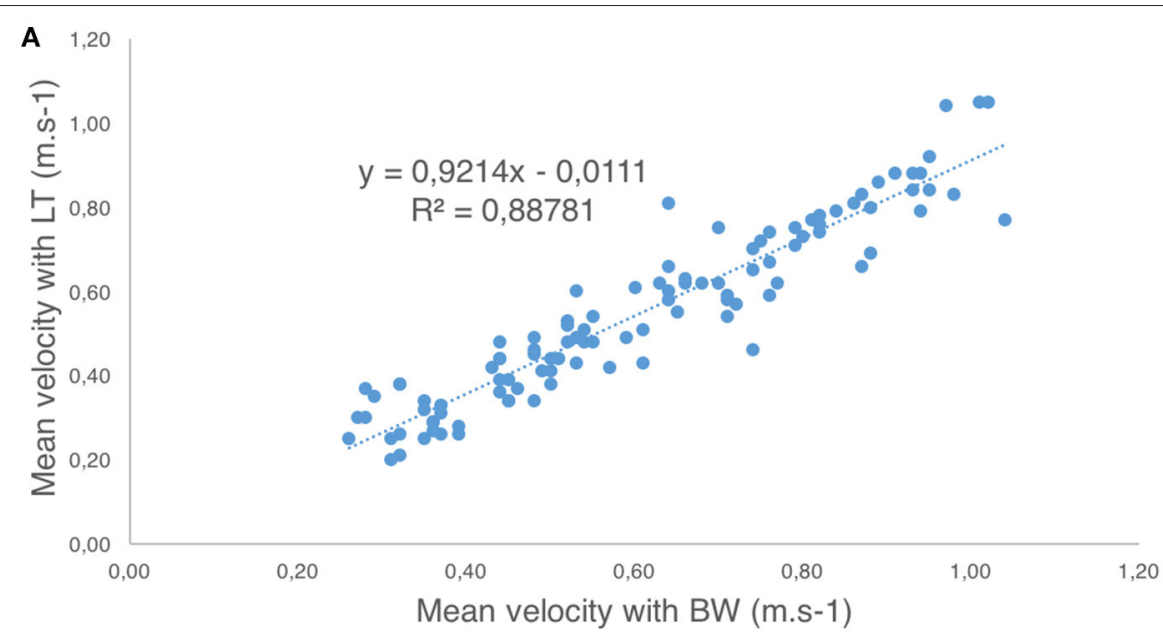

B

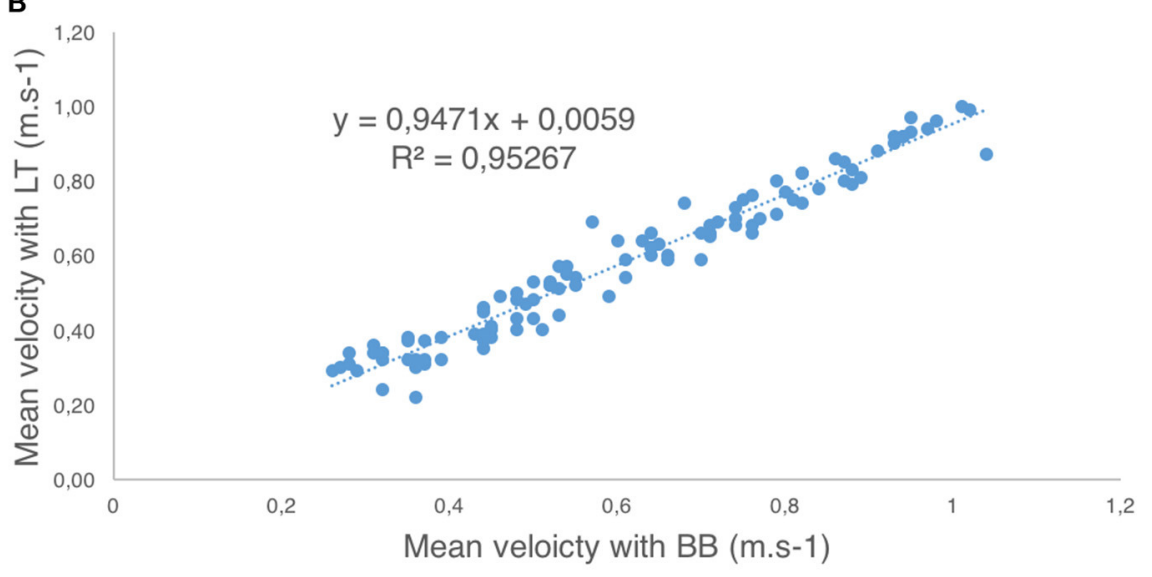

C

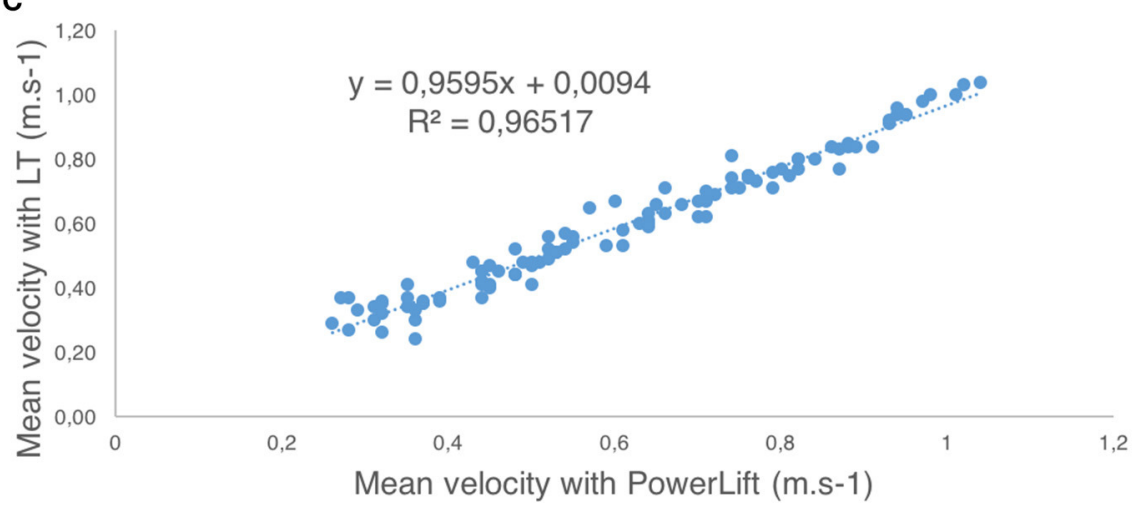

FIGURE 2 | Correlation with first order regression line between the linear transducer (LT) and: (A) Beast sensor (wrist, BW); (B) Beast sensor (barbell, BB); (C) PowerLift app for the hip-thrust exercise. 


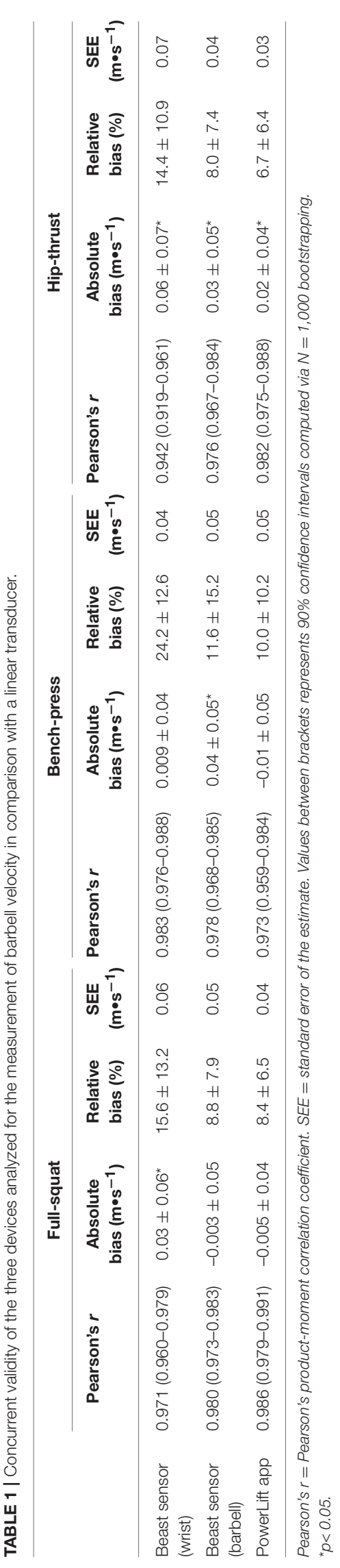

\section{Validity}

Concurrent validity was tested using Pearson's product-moment correlation coefficient (r) with $90 \%$ confidence intervals (CI) via $N=1,000$ bootstrapping. Scores from 0.8 to 0.9 were considered as good, while values above $>0.9$ were considered as high (Vincent and Weir, 2012). Moreover, the analysis of the regression line on the Bland-Altman plots was used to check for heterogeneity of the observed differences across the whole range of velocities analyzed. Finally, one way ANOVA was used to compare the load-velocity profiles between the four devices analyzed (i.e., the LT, BW, BB, and the PowerLift app).

\section{Reliability}

To test the reliability of the different devices for the measurement of the two repetitions performed on the 5 initial sets of the incremental test, the intraclass correlation coefficient (ICC) with $90 \%$ CI was used. Scores from 0.8 to 0.9 were considered as good, while values above $>0.9$ were considered as high (Vincent and Weir, 2012).

The level of significance was set at 0.01. All calculations were performed using IBM ${ }^{\circledR}$ SPSS ${ }^{\circledR}$ Statistics 22 software (IBM Co., USA).

\section{RESULTS}

\section{Validity and Accuracy}

Pearson's product-moment correlation coefficient revealed a high correlation between the values of mean velocity measured with the linear transducer and BW $(r=0.94-0.98$, SEE $=0.04-0.07$ m॰s $\left.{ }^{-1}\right)$, BB $\left(r=0.97-0.98\right.$, SEE $\left.=0.04-0.05 \mathrm{~m} \bullet \mathrm{s}^{-1}\right)$, and the PowerLift app $\left(r=0.97-0.98\right.$, SEE $\left.=0.03-0.05 \mathrm{~m} \bullet \mathrm{s}^{-1}\right)$ for the three exercises analyzed. See Figure 2.

When analyzing the accuracy of the Beast sensor and the PowerLift app for the measurement of barbell velocity in comparison with the LT, systematic biases where observed for BW, BB, and the app in the hip-thrust and the bench-press exercise, and for BW in the full-squat exercise $(p<0.001)$, with values of the LT being systematically higher in most cases [BW (mean difference $=0.01-0.06 \mathrm{~m} \bullet \mathrm{s}^{-1}$ ); BB (mean difference $=0.03-0.04 \mathrm{~m}^{-1}$ ); PowerLift app (mean difference $=-0.01$ to $0.02 \mathrm{~m} \bullet \mathrm{s}^{-1}$ )]. See Table 1 for more details. Moreover, differences between the LT and each device were similar across the whole range of velocities analyzed as revealed by the linear regression of the Bland-Altman plots (BW: $R^{2}=0.004-$ 0.03, BB: $R^{2}=0.007-0.01$, PowerLift: $\left.R^{2}=0.001-0.03\right)$. See Figure 3.

\section{Reliability}

When analyzing the two repetitions performed with each load ranging 50-95\% 1-RM, high agreements were observed for all the devices and exercises analyzed (ICC > 0.9). Moreover, the levels of reliability of the BW, BW, and the PowerLift app were very similar to those observed with the LT. See Table 2 for more details. 
A

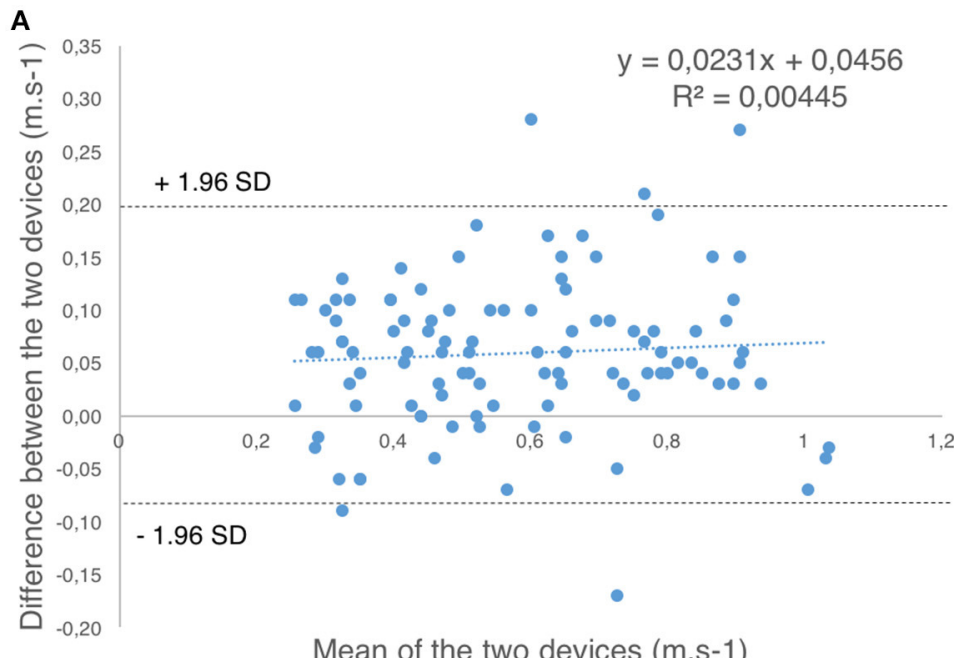

Mean of the two devices (m.s-1)

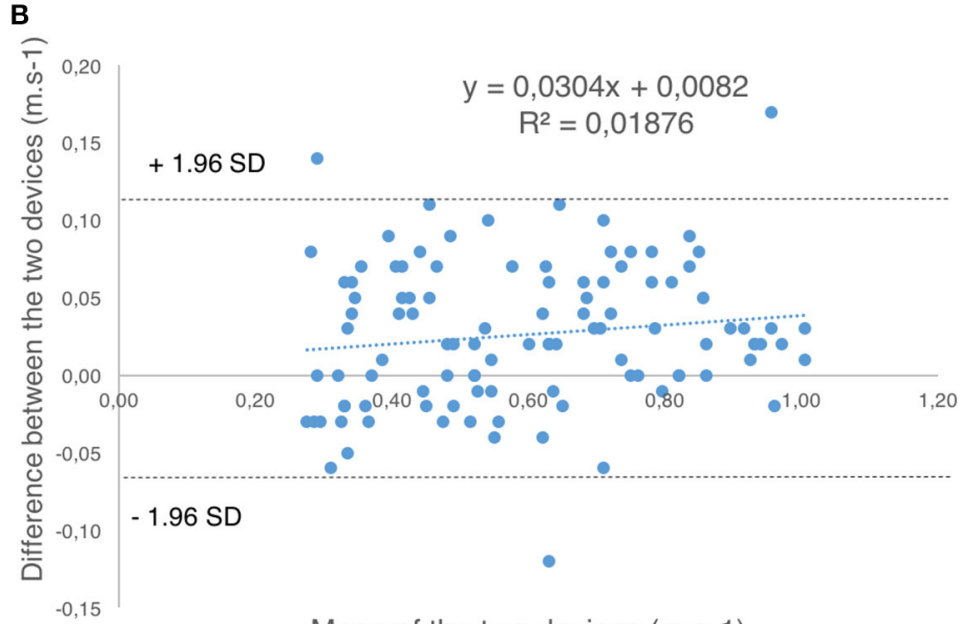

Mean of the two devices (m.s-1)

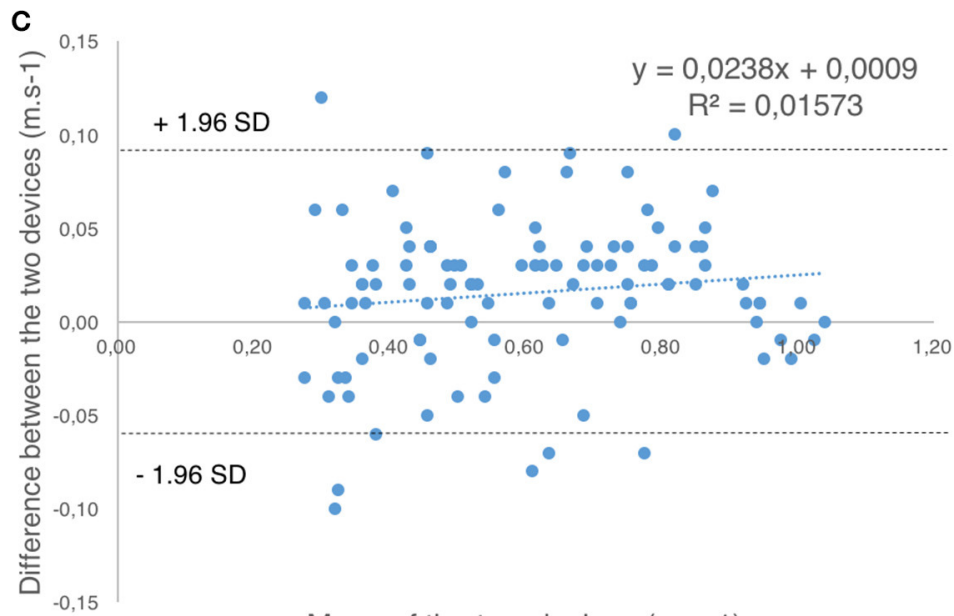

Mean of the two devices (m.s-1)

FIGURE 3 | Bland-Altman plots for the measurement of barbell velocity between the linear transducer (LT) and: (A) Beast sensor (wrist, BW); (B) Beast sensor (barbell, BB); (C) PowerLift app for the hip-thrust exercise. The blue dashed line represents the first-order regression line of the data, while the gray dashed lines represents \pm 1.96 standard deviations $(S D)$. 
TABLE 2 | Intraclass correlation coefficient for the measurement of the two repetitions performed with loads ranging 50-95\% 1-RM with the three exercises, for all the devices analyzed.

\begin{tabular}{lccc}
\hline & Full-squat & Bench-press & Hip-thrust \\
\hline Linear & 0.981 & 0.981 & 0.966 \\
transducer & $(0.965-0.990)$ & $(0.965-0.990)$ & $(0.937-0.982)$ \\
Beast sensor & 0.975 & 0.977 & 0.952 \\
(wrist) & $(0.955-0.986)$ & $(0.958-0.988)$ & $(0.910-0.974)$ \\
Beast sensor & 0.979 & 0.981 & 0.958 \\
(barbell) & $(0.962-0.988)$ & $(0.966-0.990)$ & $(0.922-0.977)$ \\
PowerLift app & 0.981 & 0.974 & 0.961 \\
& $(0.965-0.989)$ & $(0.951-0.986)$ & $(0.928-0.979)$ \\
\hline
\end{tabular}

ICC $=$ Intraclass correlation coefficient. Values between brackets represents $90 \%$ confidence intervals computed via $N=1,000$ bootstrapping.

\section{Load-Velocity Profile}

When analyzing the individual load-velocity relationships of the three studied exercises using least-squares regression, no significant differences were observed in the slope, intercept or $R^{2}$ values $(p>0.05)$ computed with the velocities measured with the LT, BW, BB, or PowerLift app as revealed by the one-way ANOVA. See Figure 4 for more details.

\section{DISCUSSION}

Both the Beast wearable device (in both of its configurations) and the PowerLift iOS app were found to have acceptable validity and reliability in comparison with a linear transducer (LT) for the measurement of barbell velocity in the full-squat, bench-press, and hip-thrust exercises. Specifically, bootstrapping analysis $(N=1,000)$ showed a very narrow confidence interval for Pearson's product-moment correlation coefficient $(r=$ 0.94-0.98) for the three devices, highlighting the high level of agreement between BW, BB, PowerLift, and the LT for the measurement of barbell velocity. Moreover, although systematic biases were observed for velocities measured with BW, BB, and the app in the hip-thrust and bench-press exercises, the absolute difference was small in all cases (mean difference $=-0.01$ to $0.06 \mathrm{~m}^{-1}$ ) and, furthermore, the analysis of the regression lines of the Bland-Altman plots showed that differences between the novel devices and the gold standard were homogeneous across all the ranges of velocities measured $\left(R^{2}=0.001-0.03\right)$. These results are in line with previous research that showed very similar validity, reliability, and accuracy scores for the measurement of barbell velocity in the bench-press exercise using the PowerLift app (Balsalobre-Fernández et al., 2017) and confirms the suitability of high-speed video analysis to assess different activities such as jumping, sprinting, running, or lifting (Balsalobre-Fernández et al., 2015, 2016a; Romero-Franco et al., 2016; Sañudo et al., 2016). To the best of our knowledge, no previous studies have analyzed the Beast sensor for the measurement of movement velocity in resistance exercises; however, other wearable devices designed for the same purpose were recently studied (Balsalobre-Fernández et al., 2016b; Banyard et al., 2017). Specifically, the PUSH wearable device also showed high levels of validity and reliability in comparison with a linear transducer $(r>0.9)$ for the measurement of movement velocity (Balsalobre-Fernández et al., 2016b). However, results in our study showed that the Beast sensor, both attached to the barbell or to the wristband, has superior values of correlation and accuracy in comparison with a linear transducer working at $1 \mathrm{kHz}$. While the correlation between the PUSH band and a $1 \mathrm{kHz}$ linear transducer was good for the measurement of back-squat $(r=0.86)$ in the mentioned study, this value is remarkably lower than those obtained both with $\mathrm{BW}$ and $\mathrm{BB}$ in our study for the same exercise ( $r=0.960-0.983)$, meaning that the Beast sensor seems to be better associated with the measures obtained with a $1 \mathrm{kHz}$ LT. Moreover, although both devices showed homogeneous differences across the whole range of velocities analyzed in comparison with the LT (as revealed by the Bland-Altman plots), the accuracy of the Beast sensor was also superior that the one observed with the PUSH band $\left(0.01,0.06\right.$, and $0.11 \mathrm{~m}^{-1}$ of mean difference with respect to $\mathrm{LT}$ for $\mathrm{BB}, \mathrm{BW}$, and $\mathrm{PUSH}$ band respectively). These differences could be in part because each wearable is designed to be placed in different body parts. While the Beast sensor is designed to be placed in a wristband or directly attached to the barbell, the PUSH band is meant to be placed just below the elbow of the subject. Therefore, the Beast sensor is much closer to the barbell than the PUSH band, which could provide better measures in comparison with a LT. However, to better compare these two wearables for the measurement of barbell velocity, more studies should be conducted measuring the exact same repetitions with respect to the same LT.

Another main result in our study was the comparison of the load-velocity profiles derived from the velocities measured with BW, BB, the PowerLift app, and the LT. The measurement of the load-velocity profiles (using a first-order linear regression fit) is of great interest since it describes the ability of the subject to produce velocity at loads of increasing intensity and it is used to estimate the 1-RM and each of its percentages with high accuracy without the need to conduct an actual 1-RM test (Jidovtseff et al., 2011; Picerno et al., 2016; Muñoz-López et al., 2017). Several studies have analyzed the load-velocity profiles on different exercises such as fullsquat (Conceição et al., 2016), bench-press (Gonzalez-Badillo and Sánchez-Medina, 2010) or pull-up (Muñoz-López et al., 2017) and it was showed, in all cases, that the load (in terms of \%1-RM) is highly correlated with the velocity at which each load is moved. Therefore, the analysis of the loadvelocity profiles is getting an increasing interest in strength and conditioning and is the basis of the recent interest on the socalled velocity-based resistance training (Gonzalez-Badillo and Sánchez-Medina, 2010; González-Badillo et al., 2015; MuñozLópez et al., 2017). In this sense, we compared the slopes and $y$-intercepts of the load-velocity profiles computed with the velocities of BW, BB, the PowerLift app, and the LT to test if similar values were obtained. One way ANOVA showed no significant differences between BW, BB, or the PowerLift app and the LT $(p>0.05)$ for the three exercises analyzed. Moreover, the four regression lines are practically 

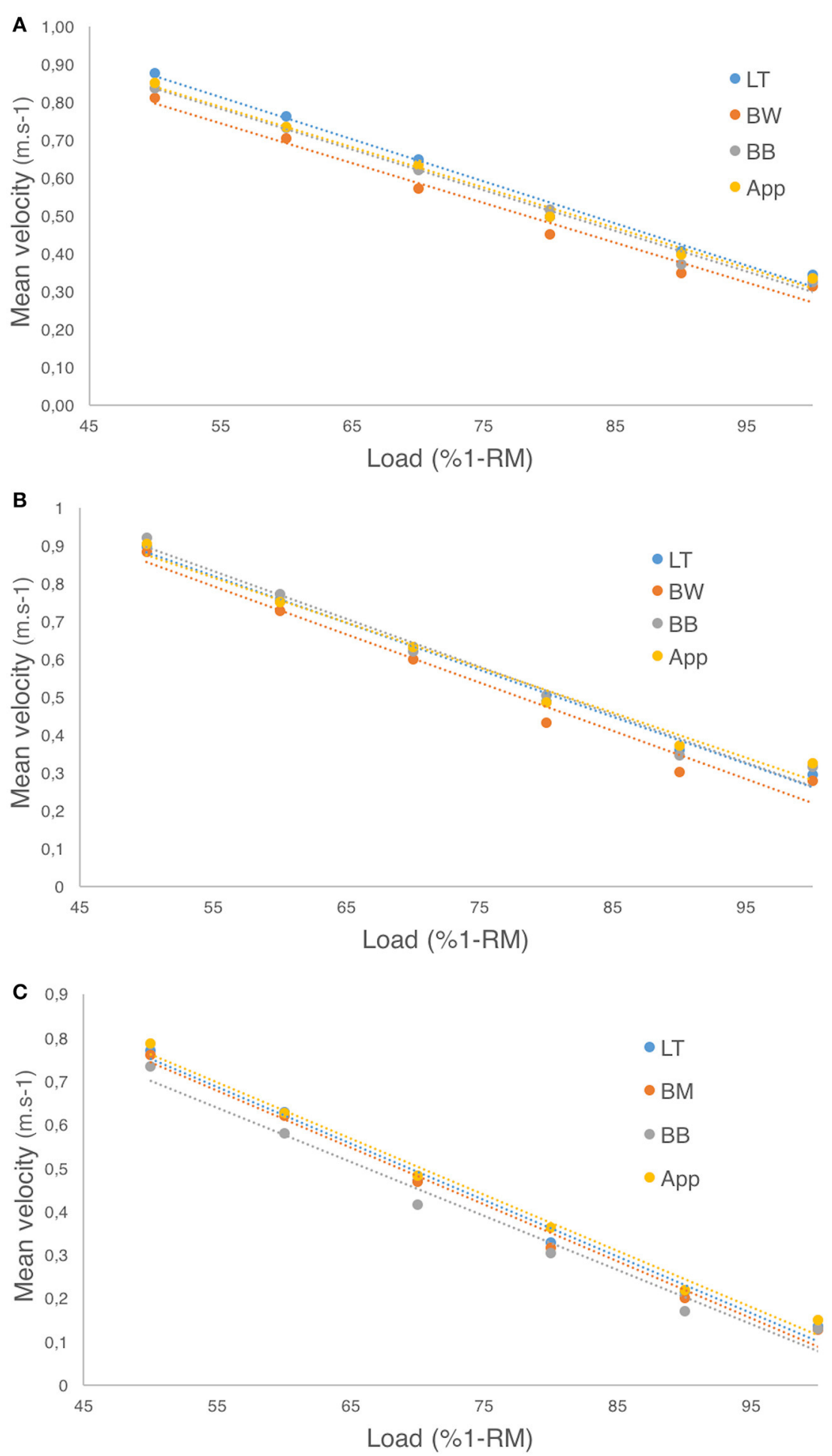

FIGURE 4 | Load-velocity profiles computed from velocities obtained by each device for: (A) Hip-thrust; (B) Full-squat; (C) Bench-press. App: PowerLift app; BW = Beast sensor (wrist); BB = Beast sensor (barbell); LT = linear transducer.

overlapped when they are plotted in the same graph (see Figure 4). Therefore, both the Beast sensor (in the wrist or in the barbell) and the PowerLift app are showed to provide accurate estimations of the load-velocity profiles of the subject and, consequently, can be used to estimate the 1-RM of the subjects. 
It is worth noting that, while BB, BW, and the app had similar values of validity, reliability and accuracy for the measurement of barbell velocity with respect to the LT, the Beast sensor provided slightly better results in all tests when it was attached to the barbell in comparison with wearing it on the wrist. Therefore, although the Beast sensor is categorized as a wearable device, our results suggest that it not should be worn in the wrist, but attached to the barbell in order to get the more accurate results.

In conclusion, the Beast sensor (both in the wrist or attached to the barbell) and the PowerLift app were showed to be highly, valid, reliable, and accurate for the measurement of barbell velocity in the full-squat, bench-press, and hip-thrust exercises in comparison with a linear transducer. Moreover, these devices

\section{REFERENCES}

Balsalobre-Fernández, C., Agopyan, H., and Morin, J.-B. (2016a). The validity and reliability of an iphone app for measuring running mechanics. J. Appl. Biomech. 33, 222-226. doi: 10.1123/jab.2016-0104

Balsalobre-Fernández, C., Glaister, M., and Lockey, R. A. (2015). The validity and reliability of an iPhone app for measuring vertical jump performance. J. Sports Sci. 33, 1574-1579. doi: 10.1080/02640414.2014. 996184

Balsalobre-Fernández, C., Kuzdub, M., Poveda-Ortiz, P., and Del CampoVecino, J. (2016b). Validity and reliability of the PUSH wearable device to measure movement velocity during the back squat exercise. J. Strength Cond. Res. 30, 1968-1974. doi: 10.1519/JSC.000000000 0001284

Balsalobre-Fernández, C., Marchante, D., Muñoz-López, M., and Jiménez, S. L. (2017). Validity and reliability of a novel iPhone app for the measurement of barbell velocity and 1RM on the bench-press exercise. J. Sports Sci. 18, 1-7. doi: 10.1080/02640414.2017.1280610

Banyard, H. G., Nosaka, K., and Haff, G. G. (2016). Reliability and validity of the load-velocity relationship to predict the 1RM back squat. J. Strength Cond. Res. 31, 1897-1904. doi: 10.1519/JSC.0000000000 001657

Banyard, H. G., Nosaka, K., Sato, K., and Haff, G. G. (2017). Validity of various methods for determining velocity, force and power in the back squat. Int. J. Sports Physiol. Perform. 9, 1-25. doi: 10.1123/ijspp.20 16-0627

Borresen, J., and Lambert, M. I. (2009). The Quantification of training load, the training response and the effect on performance. Sport Med. 39, 779-795. doi: 10.2165/11317780-000000000-00000

Buckner, S. L., Jessee, M. B., Mattocks, K. T., Mouser, J. G., Counts, B. R., Dankel, S. J., et al. (2017). Determining strength: a case for multiple methods of measurement. Sport Med. 47, 193-195. doi: 10.1007/s40279-016-0 580-3

Conceição, F., Fernandes, J., Lewis, M., Gonzaléz-Badillo, J. J., and Jimenéz-Reyes, P. (2016). Movement velocity as a measure of exercise intensity in three lower limb exercises. J. Sports Sci. 34, 1099-1106. doi: 10.1080/02640414.2015.1090010

Folland, J. P., and Williams, A. G. (2007). The adaptations to strength training. Sport Med. 37, 145-168. doi: 10.2165/00007256-20073702 $0-00004$

González-Badillo, J. J., Pareja-Blanco, F., Rodríguez-Rosell, D., Abad-Herencia, J. L., Del Ojo-López, J. J., and Sánchez-Medina, L. (2015). Effects of velocity-based resistance training on young soccer players of different ages. J. Strength Cond. Res. 29, 1329-1338. doi: 10.1519/JSC.0000000000 000764

Gonzalez-Badillo, J. J., and Sánchez-Medina, L. (2010). Movement velocity as a measure of loading intensity in resistance training. Int. J. Sports Med. 31, 347-352. doi: 10.1055/s-0030-1248333 were proven to be highly suitable for the analysis of the loadvelocity profiles in the mentioned exercises. These results could have potential interest for strength and conditioning coaches who wish to monitor movement velocity in the full-squat, benchpress, and hip-thrust exercises.

\section{AUTHOR CONTRIBUTIONS}

I confirm that all authors have significantly contributed to this manuscript. The specific tasks developed by each researcher were as follows: $\mathrm{CB}, \mathrm{DM}, \mathrm{MM}$, and SJ: design of the experiment. DM, MM, EB, and IA: data collection. CB, SJ, and MM: writing of the manuscript. CB: statistical analyses. All authors: revision of the manuscript.

Halson, S. (2016). Wearable technology for athletes : information overload and pseudoscience ? Int. J. Sport Physiol. Perform. 11, 705-706. doi: 10.1123/IJSPP.2016-0486

Jidovtseff, B., Harris, N. K., Crielaard, J.-M., and Cronin, J. B. (2011). Using the load-velocity relationship for $1 \mathrm{RM}$ prediction. J. Strength Cond. Res. 25, 267-270. doi: 10.1519/JSC.0b013e3181b $62 \mathrm{c} 5 \mathrm{f}$

Kravitz, L., Akalan, C., Nowicki, K., and Kinzey, S. J. (2003). Prediction of 1 repetition maximum in high-school power lifters. J. Strength Cond. Res. 17, 167-172.

Muñoz-López, M., Marchante, D., Cano-Ruiz, M. A., Chicharro, J. L., and Balsalobre-Fernández, C. (2017). Load, force and power-velocity relationships in the prone pull-up exercise. Int. J. Sports Physiol. Perform. 2, 1-22. doi: 10.1123/ijspp.2016-0657

Naclerio, F., and Larumbe-Zabala, E. (2016). Relative load prediction by velocity and the omni-res $0-10$ scale in parallel squat. J. Strength Cond. Res. 31, 1585-1591. doi: 10.1519/JSC.00000000000 01601

Pareja-Blanco, F., Rodríguez-Rosell, D., Sánchez-Medina, L., Sanchis-Moysi, J., Dorado, C., Mora-Custodio, R., et al. (2016). Effects of velocity loss during resistance training on athletic performance, strength gains and muscle adaptations. Scand. J. Med. Sci. Sports 27, 724-735. doi: 10.1111/sms. 12678

Picerno, P., Iannetta, D., Comotto, S., Donati, M., Pecoraro, F., Zok, M., et al. (2016). 1RM prediction: a novel methodology based on the forcevelocity and load-velocity relationships. Eur. J. Appl. Physiol. 116, 2035-2043. doi: 10.1007/s00421-016-3457-0

Robertson, R. J., Goss, F. L., Aaron, D. J., Gairola, A., Kowallis, R. A., Ying, L., et al. (2008). One repetition maximum prediction models for children using the omni rpe scale. J. Strength Cond. Res. 22, 196-201. doi: 10.1519/JSC.0b013e31815f6283

Romero-Franco, N., Jiménez-Reyes, P., Castaño-Zambudio, A., Capelo-Ramírez, F., Rodríguez-Juan, J. J., González-Hernández, J., et al. (2016). Sprint performance and mechanical outputs computed with an iPhone app: Comparison with existing reference methods. Eur. J. Sport Sci. 17, 1-7. doi: 10.1080/17461391.2016.1249031

Sánche-Medina, L., and Gonzalez-Badillo, J. J. (2011). Velocity loss as an indicator of neuromuscular fatigue during resistance training. Med Sci. Sport Exerc. 43, 1725-1734. doi: 10.1249/MSS.0b013e31821 $3 \mathrm{f} 880$

Sánchez-Medina, L., Gonzalez-Badillo, J. J., Perez, C. E., and Pallares, J. G. (2014). Velocity and power-load relationships of the bench pull vs. bench press exercises. Int. J. Sports Med. 35, 209-216. doi: 10.1055/s-0033-13 51252

Sañudo, B., Rueda, D., Pozo-Cruz, B., Del de Hoyo, M., and Carrasco, L. (2016). Validation of a video analysis software package for quantifying movement velocity in resistance exercises. J. Strength Cond. Res. 30, 2934-2941. doi: 10.1519/JSC.0000000000000563 
Thompson, W. R. (2016). Worldwide survey of fitness trends for 2017. ACSM's Heal Fit. Trends. 20, 8-17. doi: 10.1249/FIT.0000000000 000252

Tillin, N., and Folland, J. (2014). Maximal and explosive strength training elicit distinct neuromuscular adaptations, specific to the training stimulus. Eur. J. Appl. Physiol. 114, 365-374. doi: 10.1007/s00421-013-2 781-x

Tufano, J. J., Conlon, J. A., Nimphius, S., Brown, L. E., Seitz, L. B., Williamson, B. D., et al. (2016). Maintenance of velocity and power with cluster sets during high-volume back squats. Int. J. Sports Physiol. Perform. 11, 885-892. doi: 10.1123/ijspp.2015-0602

Vincent, W., and Weir, J. P. (2012). Statistics in Kinesiology, 4th Edn. Champaign, IL: Human Kinetics.

Zourdos, M. C., Klemp, A., Dolan, C., Quiles, J. M., Schau, K. A., Jo, E., et al. (2015). Novel resistance training-specific RPE scale measuring repetitions in reserve. J. Strength Cond. Res. 30, 267-75. doi: 10.1519/JSC.0000000000001049
Conflict of Interest Statement: The first author of the article (CB) is the developer of the app mentioned. To guarantee the independency of the data collection, the data from the app were obtained by an independent researcher (IA), who has no personal or professional relationship with $\mathrm{CB}$, and is not related to the app in any way. The other three authors who were in charge of the data collection as reported in the author contribution statement registered data from the other three devices analyzed in this study.

Copyright (๔) 2017 Balsalobre-Fernández, Marchante, Baz-Valle, Alonso-Molero, Jiménez and Muñóz-López. This is an open-access article distributed under the terms of the Creative Commons Attribution License (CC BY). The use, distribution or reproduction in other forums is permitted, provided the original author(s) or licensor are credited and that the original publication in this journal is cited, in accordance with accepted academic practice. No use, distribution or reproduction is permitted which does not comply with these terms. 